\title{
Region Of Interest Based Image Classification Using Time Series Analysis
}

\author{
A. Elsayed, F. Coenen, M. García-Fiñana and V. Sluming
}

\begin{abstract}
An approach to Region Of Interest Based Image Classification (ROIBIC), based on a time series analysis approach, is described. The focus of the approach is the classification of MRI brain scan data according to the nature of the corpus callosum (a feature within such scans), however the approach also has general applicability. The advocated approach combines a number of image processing techniques combined with time series analysis, specifically dynamic time warping. Of note is the mechanism used to generate the desired time series. The application of the time series based ROIBIC demonstrates that the proposed approach performs both efficiently and effectively, obtaining a classification accuracy of over $98 \%$ in the case of the given application. Comparisons are also presented with a graph based ROIBIC approach.
\end{abstract}

\section{INTRODUCTION}

Image classification typically involves the preprocessing of collections of images into a format whereby established classification techniques may be applied. Typical applications include: cerebral morphology [1], remote sensing image classification [2] and content based medical image retrieval [3]. As with many data mining applications the main challenge in the preprocessing of image data is to produce a representation whereby no relevant information is lost, however the end result is succinct enough to allow efficient data mining. In image data mining this challenge is more acute because it is usually not clear what can be thrown away. In most cases it is not possible to preprocess the images in such a way that all pixel data is retained - some decision regarding resolution needs to be made. The most straight forward approach is to apply some form of segmentation to identify "blobs" within the images and then use these blobs as an attribute set. Some further background discussion regarding these issues associated with image mining, and particularly medical image classification, are presented in Section II.

This paper describes an approach to image classification whereby the classification is conducted according to the size and shape of a region of interest that occurs across the image set, we refer to this as Region Of Interest Based Image Classification (ROIBIC). This approach is of course

Ashraf Elsayed is with the Department of Computer Science, University of Liverpool, Ashton Building, Ashton Street, Liverpool L69 3BX, United Kingdom (email: a.el-sayed@liv.ac.uk).

Frans Coenen is with the Department of Computer Science, University of Liverpool, Ashton Building, Ashton Street, Liverpool L69 3BX, United Kingdom (email: coenen@liv.ac.uk).

Marta García-Fiñana is with the Centre for Medical Statistics and Health Evaluation, University of Liverpool, Shelleys Cottage, Brownlow Street, Liverpool L69 3GS, United Kingdom (email: m.garciafinana@liv.ac.uk).

Vanessa Sluming is with the School of Health Sciences, Thompson Yates Building, The Quadrangle, Brownlow Hill, Liverpool L69 3GB, United Kingdom (email: vanessa.sluming@liv.ac.uk). not applicable to all kinds of image data, however it does have common application in medical image analysis where we are often interested in the nature of a particular element in the image set. The exemplar application, in the context of this paper, is MRI brain scan data where we wish to classify such images according to a particular element within such scans, namely the corpus callosum. There are many similar applications in medical image analysis.

The advantage of ROIBIC, i.e. focussing on a particular element within the image set, is that the storage requirements for the image representation can be significantly reduced and consequently the representation can be more detailed. To identify the feature of interest the application of segmentation techniques is still required, and this will entail the established difficulties encountered when conducting this process (i.e. poor image contrast, intensity inhomogeneities and partialvolume effects). These issues are exacerbated in the context of MRI scan image data, because these images tend to display a significant amount of noise; and in the context of the particular application featured in this paper, because the intensity-level distributions between different soft tissues are not widely distributed, and moreover the complexity of tissue boundaries cause many pixels to contain mixtures of tissues. For the identification of the corpus callosum the authors make use of Felzenszwalb and Huttenlocher's graph-based segmentation algorithm [4], which is described in more detail in Section III.

Once the feature of interest has been identified this can be described in a variety of ways depending on the nature of the classification mechanism to be applied. The authors have experimented with a variety of representation mechanisms for ROIBIC, all designed to retain the shape and size information associated with the feature of interest. This paper reports a time series representation for ROIBIC to which a time series analysis approach to classification can be applied. The particular time series analysis approach used is a Dynamic Time Warping (DTW) technique as first proposed by Berndt and Clifford [5]. The specific time series generation process advocated in this paper is described in Section IV, and the DTW based classification mechanism in section V. The overall ROIBIC methodology advocated in this paper is detailed in Section VI. The authors have also experimented with graph representations for ROIBIC [6], some details are included in the previous work section of this paper (Section II.), and some comparisons are made between the two techniques in the evaluation section (Section VII).

As noted above, the focus of the work described here is 
brain MRI scans. The region of interest in these images is called the corpus callosum. An example image is given in Figure 1. The corpus callosum is located in the centre of the image, the fornix is a related structure which often "blurs" into the corpus callosum and presents a particular challenge in the context of segmentation. The corpus callosum is of interest to medical researchers for a number of reasons. The size and shape of the corpus callosum have been shown to be correlated to sex, age, neurodegenerative diseases (such as epilepsy) and various lateralized behaviour in people. It is also conjectured that the size and shape of the corpus callosum reflects certain human characteristics (such as a mathematical or musical ability).

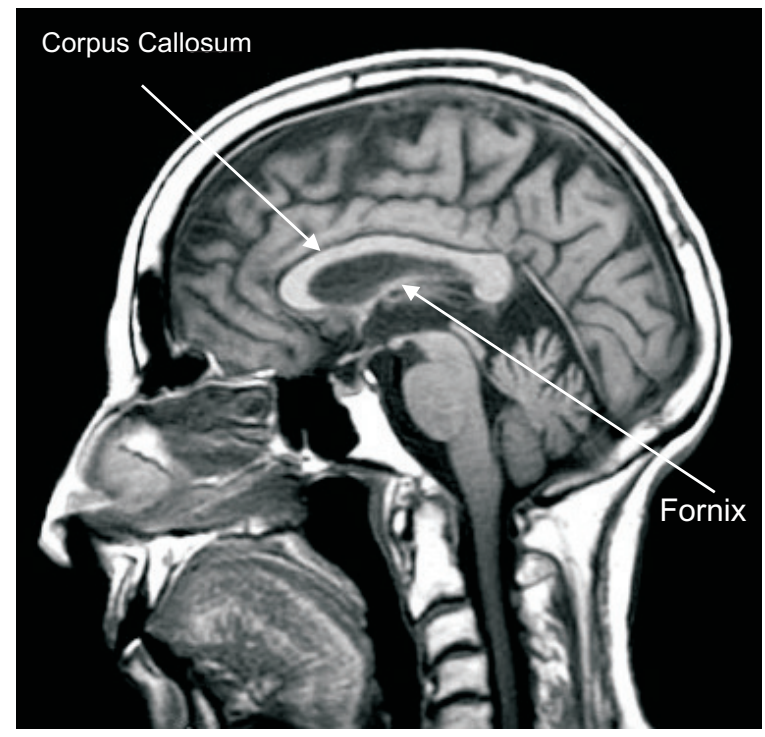

Fig. 1. corpus callosum in a midsagittal brain MR image.

The main contributions of the work described here may be summarised as follows: (i) an alternative approach to image classification, where the focus is on a particular region of interest occurring across the image set, which we have termed Region Of Interest Based Image Classification (ROIBIC), (ii) an interesting and inventive approach to representing such features as a time series and (iii) a novel application of time series analysis for ROIBIC.

\section{PREVIOUS WORK}

The main challenge of image mining, as noted above, is the appropriate preprocessing of the data so that established data mining techniques can be applied. There are a variety of ways in which images can be processed, but the most common technique is to apply some form of segmentation (alternative approaches include the use of histograms, vector based representations or some form of pixel representation). Segmentation refers to the process of partitioning a digital image into multiple segments (sets of pixels). The goal of segmentation is to simplify and/or change the representation of an image into something that is more meaningful and easier to analyse. Image segmentation is typically used to locate objects and boundaries (lines, curves, etc.) in images. In the context of the work described in this paper, image segmentation plays a role in the identification of the corpus callosum that may feature within brain MRI scans. More details concerning the image segmentation process used is presented in Section III. The main challenge of automatic brain MRI scan segmentation is the absence of distinct boundaries between different brain tissues. This is because of various reasons: firstly medical MR images have a lot of noises, secondly in MR images the intensity-level distributions between different soft tissues are not widely distributed and moreover the complexity of tissue boundaries causes many pixels to contain mixtures of tissues.

Classification involves the construction of a classifier using pre labelled data (the training set), which can then be applied to "unseen" data (the test set) so as to classify the records in this unseen data. There are many established techniques for building classifiers from a given training set, common examples include: decision trees [7], [8], neural networks [9], [10], support vector machines [11], [12] and rule based classifiers [14], [15]. The accuracy of a classifier is usually established by applying it to the test set. A different approach, and that used in this paper, is where the training set defines a set of "prototypes" which, coupled with a similarity engine, is used to classify unseen data. The approach has strong correlations with Case Based Reasoning (CBR) as described in [16], [17], [18], consequently we refer to the set of prototypes as the case base.

With respect to the ROIBIC approach described in this paper the case base prototypes are described in terms of time series. A time series is an ordered sequence of values of a variable, sampled at some temporal interval. Time series analysis comprises methods for analysing time series data in order to extract meaningful statistics and other characteristics of the data. One approach to time series analysis makes use of a variation of Dynamic Time Warping (DTW) [5]. DTW is a time series comparison technique that allows "shifting" in the time domain. In other words DTW minimizes the effect of shifting in time by allowing the transformation of time series in order to detect similar shapes with different phases. DTW also breaks the limitation of one-to-one alignment, and also supports non-equal-length time series. More details concerning the DTW technique incorporated into the proposed time series ROIBIC is presented in Section V. In the approach described DTW has been adopted as the similarity engine to be applied to the image case base.

An alternative ROIBIC representation to the proposed in this paper, that the authors have experimented with, is a graph based representation [6]. This is described briefly in the following sub-section. In order to analyse the time series ROIBIC approach a comparison with this graph based approach is presented in section VII. 


\section{A. A Graph Based Approach}

ROIBIC using a grpah based representation was introduced by the authors in [6]. As in the case of this paper, the work was directed at the classification of MRI images according to the corpus callosum feature. The classification process commenced with the segmentation of the input images. A registration process was then applied, so that each identified corpus callosum was founded upon the same origin. The pixel representation, for each corpus callosum, was then tessellated and stored in a quad-tree data structure, one tree per image. The advantage offered by the quad tree representation, unlike some other representations, was that information about the spatial relationships between individual pixels was maintained. These trees were then processed to identify frequent sub-trees that occured across the image set. For this purpose a weighted graph mining algorithm was developed to take into consideration the greater significance that should be assigned to quad-tree nodes closer to the root than nodes further away. The identified sub-trees then formed the fundamental elements of a feature space. Each image was then represented in terms of this feature space using individual feature vectors. From experimentation it was discovered that the number of features (frequent sub-trees) could be substantial and that many features seemed to play no part in the resulting classification. To reduce the number of features to a more manageable number a feature selection mechanism was applied. The application of the decision tree algorithm then yielded the final classifier. The results demonstrated that the proposed approach performed both efficiently and effectively, obtaining a classification accuracy of over $95 \%$ in the case of the given application. This graph based approach to ROIBIC is therefore used in the context of this paper to evaluate the proposed time series approach.

\section{SEGMENTATION}

The objective of image segmentation is to partition images into meaningful regions. For the work described here Felzenszwalb and Huttenlocher's segmentation algorithm was used [4]. This method is based on Minimum Spanning Trees (MSTs). All pixels of the original image are viewed as separate components. Two components are merged if the external variation between the components is small compared to their internal variations. External and internal variations values are based on the MST of the components. The key to the success of this method is adaptive thresholding. More specifically, let $G=(V, E)$ be a fully connected graph consisting of a set of $n$ vertices $V$ and a set of $m$ edges $E$. Each edge $e=\left(v_{i}, v_{j}\right) \in E$ has a corresponding weight $w\left(\left(v_{i}, v_{j}\right)\right)$, which is a non-negative measure of the dissimilarity between neighbouring vertices $v_{i}$ and $v_{j}$, where $v_{i}, v_{j} \in V$ and $\left\|v_{i}-v_{j}\right\| \leq d$. If $d=1$, then every vertex is connected to its immediate eight neighbours. With $d=2$, every vertex is connected to its 24 neighbours. Each vertex is a pixel, $v$, represented in the image. The corresponding weight function between two vertices can be written as $w\left(\left(v_{i}, v_{j}\right)\right)=\left|I\left(v_{i}\right)-I\left(v_{j}\right)\right|$ where $I\left(v_{i}\right)$ represents the image intensity at the vertex $v_{i}$. The final segmentation will be $S=\left(C_{1}, \ldots, C_{r}\right)$ where $C_{i}$ is a cluster of pixels. The internal variation of a component $C \subset S$ is defined as the maximum weight edge in any MST of $\mathrm{C}(M S T(C))$. Hence:

$$
\operatorname{Int}(C)=\max _{e \in M S T(C)} w(e),
$$

The minimum internal variation for components $C_{1}$ and $C_{1}$ is defined by:

$\operatorname{MInt}\left(C_{1}, C_{2}\right)=\min \left(\operatorname{Int}\left(C_{1}\right)+\tau\left(C_{1}\right), \operatorname{Int}\left(C_{2}\right)+\tau\left(C_{2}\right)\right)$,

where the threshold function $\tau$ is given by,

$$
\tau(C)=\frac{k}{\left|V^{\prime}\right|} .
$$

Here $k$ is a positive integer, and $\left|V^{\prime}\right|$ is the number of vertices in $V^{\prime}$, where $C=\left(V^{\prime}, E^{\prime}\right)$. The constant $k$ controls the degree of similarity between components and hence the number components found. Finally, the external variation between components $C_{1}=\left(V_{1}, E_{1}\right)$ and $C=\left(V_{2}, E_{2}\right)$ is defined as:

$$
\operatorname{Ext}\left(C_{1}, C_{2}\right)=\min _{v_{1} \in V_{1}, v_{2} \in V_{2}} w\left(\left(v_{i}, v_{j}\right)\right)
$$

The steps of the algorithm are as follows [4]:

1) Sort $E=\left(e_{1}, \ldots, e_{m}\right)$ such that $w\left(e_{i}\right) \leq w\left(e_{j}\right)$ for $i \leq j$.

2) Let $S^{0}=\left(\left\{v_{1}\right\}, \ldots,\left\{v_{n}\right\}\right)$, in other words each initial cluster contains exactly one vertex.

3) Repeat step 4 for $t=1, \ldots, m$

4) Construct $S^{q}$ given $S^{q-1}$ as follows. Let $v_{i}$ and $v_{j}$ denote the vertices connected by the t-th edge in the ordering. If $v_{i}$ and $v_{j}$ are in disjoint components of $S^{q-1}: C_{i}^{q-1}$ and $C_{j}^{q-1}$ respectively and $w\left(e_{t}\right) \leq$ $\operatorname{MInt}\left(C_{i}^{q-1}, C_{j}^{q-1}\right)$ then $S^{q}$ is obtained from $S^{q-1}$ by merging $C_{i}^{q-1}$ and $C_{j}^{q-1}$. Otherwise $S^{q}=S^{q-1}$.

5) Return $S=S^{m}$

\section{The TIme Series Representation}

In this section the process whereby individual time series, one per image, are generated with respect to the region of interest is presented. Broadly, the process consists of identifying the feature of interest and describing it as a time series by taking a series of measurements radiating from a fixed point located in the vicinity of the feature. The process comprises four steps: (i) data cleaning, (ii) segmentation, (iii) registration, and (iv) time series generation.

The process commences with data cleaning (noise reduction using Gaussian Smoothing). On completion of the data cleaning the segmentation was undertaken by applying Felzenszwalb and Huttenlocher's segmentation algorithm (see above), however other segmentation techniques may be equally applicable depending on the nature of the application. In the case of the MRI ROIBIC example application used in this paper the corpus callosum was identifiable because 
its approximate location was known in advance. This may not be the case for all ROIBIC applications in which case some other identification technique will have to be used. The registration process was undertaken by fitting the feature (corpus callosum) into a Minimum Bounding Rectangle (MBR). The next step was to derive the time series according to the boundary line circumscribing the feature. The time series was generated using an ordered sequence of $N$ vectors radiating out (projecting) from the midpoint of the lower edge of the MBR as shown in Figure 2. The time series was then expressed as a series of values (one for each of the $N$ vectors) describing the size (length) of intersection of the vector with the feature. This process was applied to each image in turn to produce the desired time series data set. It should be $N$ is variable depending on the length of curvature of the CC. This is why a DTW has been used as opposed to a Euclidean distance based technique. The representation of the corpus callosum as a time series can maintain the structural information of the CC.

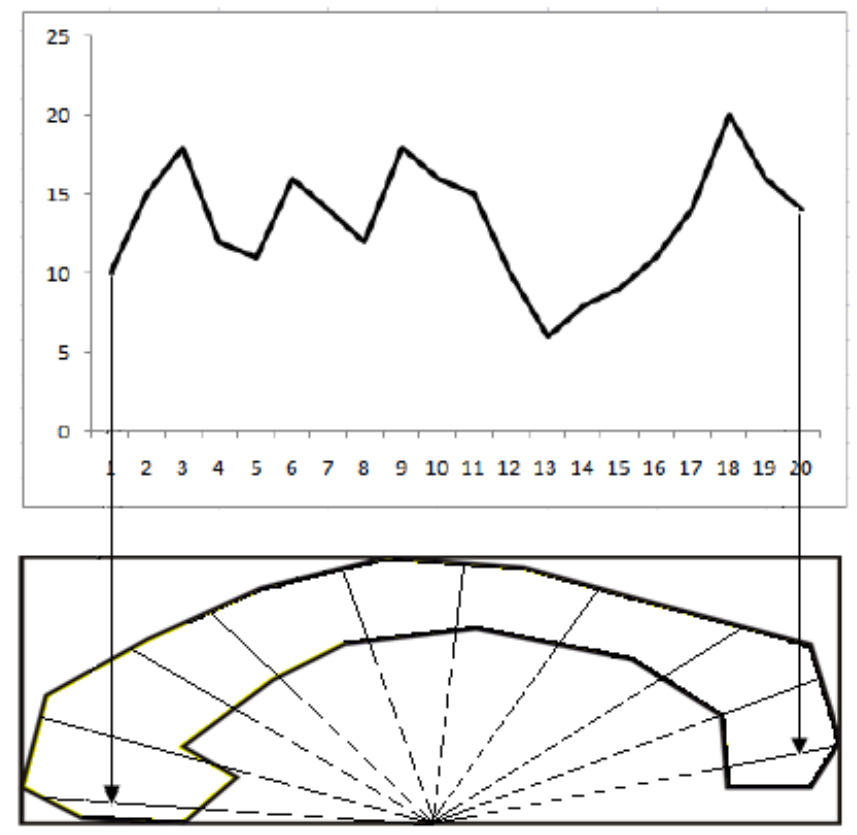

Fig. 2. Conversion of corpus callosum into time series.

\section{The Dynamic Time Warping Algorithm}

Formally, Dynamic Type Warping (DTW) may be defined as follows. Given two time series $Q$ and $C$, of length $n$ and $m$ respectively, where:

$$
\begin{gathered}
Q=q_{1}, q_{2}, \ldots, q_{i}, \ldots, q_{n} \\
C=c_{1}, c_{2}, \ldots, c_{j}, \ldots, c_{m}
\end{gathered}
$$

To align these two sequences using DTW, we construct an $n$-by- $m$ matrix where the $\left(i^{t h}, j^{t h}\right)$ elements of the matrix contains the distance $d\left(q_{i}, c_{j}\right)$ between two points $q_{i}$ and $c_{j}$ (Typically the Euclidean distance is used, so $\left.d\left(q_{i}, c_{j}\right)=\left(q_{i}, c_{j}\right)^{2}\right)$. Each matrix element $(i, j)$ corresponds to the alignment between a pair of points $q_{i}$ and $c_{j}$. This is illustrated in Figure 3. The objective is to find the minimum warping path between the two curves. A warping path, $W$, is a contiguous (in the sense stated below) set of matrix elements, $w_{k}=(i, j)_{k}$ :

$W=w_{1}, w_{2}, \ldots, w_{k}, \ldots, w_{K} \quad \max (m, n) \leq K<m+n-1$

The warping path is typically subjected to several constraints [13].

- Boundary condition: $w_{1}=(1,1)$ and $w_{K}=(m, n)$. Simply stated, this requires the warping path to start and finish in diagonally opposite corner cells of the matrix.

- Continuity: Given $w_{k}=(a, b)$ then $w_{k-1}=(a, b)$ where $a-\dot{a} \leq 1$ and $b-\dot{b} \leq 1$. This restricts the allowable steps in the warping path to adjacent cells (including diagonally adjacent cells).

- Monotonicity: $w_{k}=(a, b)$ then $w_{k-1}=(a, b)$ where $a-\dot{a} \geq 0$ and $b-b \geq 0$. This forces the points in $W$ to be monotonically spaced in time.

The number of potential warping paths that satisfy the above conditions increases exponentially with the length of the time series. However, we are interested only in paths which minimize the warping cost:

$$
\operatorname{DTW}(Q, C)=\min \left\{\sqrt{\sum_{i=1}^{K} w_{k}}\right\}
$$

This path can be found very efficiently, using dynamic programming, to evaluate the following recurrence which defines the cumulative distance $\gamma(i, j)$ as the distance $d(i, j)$ found in the current cell and the minimum of the cumulative distances of the adjacent elements:

$$
\gamma(i, j)=d\left(q_{i}, c_{j}\right)+\min \{\gamma(i-1, j-1), \gamma(i-1, j), \gamma(i, j-1)\}
$$

\section{Methodology}

In this section a brief overview is presented of the proposed region of interest based image classification (ROIBIC) methodology founded on time series analysis. The process commences with a set of pre-labelled images processed, as described above, so that each image is represented as a time series. This labelled set of time series then represents a set of proto-types held in a case base (as per the casebased reasoning paradigm). A new case is then classified according to the most similar proto-type found in the case base as identified using the DTW technique described in the foregoing Section.

\section{EVALUATION}

The focus of the advocated approach to ROIBIC using time series analysis described in this paper has been MRI scan images featuring then corpus callosum (Figure 1). This 
TABLE II
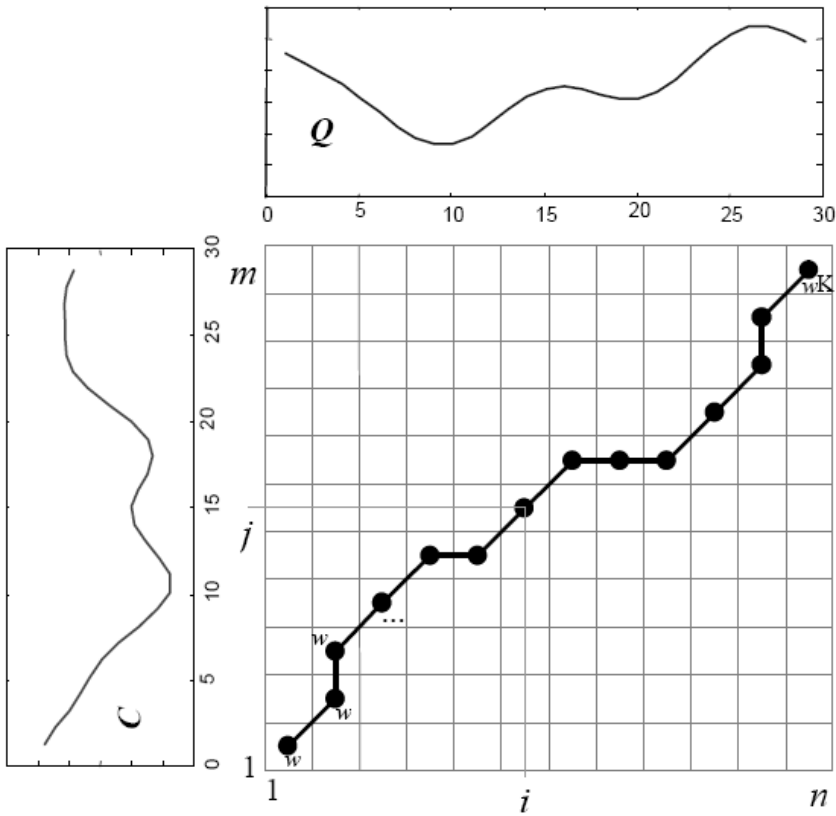

Fig. 3. An example warping path [13].

TABLE I

TCV CLASSIFICATION ACCURACY (\%) USING TIME SERIES ROIBIC

\begin{tabular}{|r|r|}
\hline Test set ID & Accuracy $(\%)$ \\
\hline 1 & 91 \\
2 & 100 \\
3 & 91 \\
4 & 100 \\
5 & 100 \\
6 & 100 \\
7 & 100 \\
8 & 100 \\
9 & 100 \\
10 & 100 \\
\hline Average & 98.2 \\
\hline SD & 3.8 \\
\hline
\end{tabular}

section describes the evaluation of the proposed technique using an appropriate MRI image set. The evaluation was undertaken in the context of classification accuracy.

The data set used comprised 106 brain images divided equally into two categories (classes), 53 images per category: musicians and non-musicians. It is acknowledged that, in data mining terms, a data set of 106 records is small. However, one of the challenges of the work described here is the limited amount of raw data available for experimentation. This is because of a number of difficulties that are presented when collecting MRI data, namely: (i) the data is expensive to produce, (ii) MRI scanning is time consuming, and (iii) ethical approval is required if they are to be used for research purposes. Consequently MRI data sets available for research purposes tend to be relatively small, compared to the usual data sets used for the evaluation of data mining techniques.

Table I shows the Ten Cross Validation (TCV) classification results (test set to training sets size ratio of between 11:95 to 10:96) obtained using our proposed technique.

For comparison purposes Table II shows the TCV clas-
TCV CLASSIFICATION ACCURACY (\%) USING GRAPH BASED ROIBIC

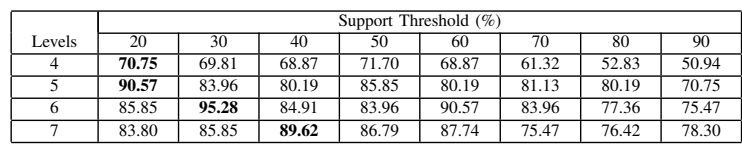

TABLE III

CLASSIFICATION ACCURACY (\%) USING TIME SERIES ROIBIC FOR DIFFERENT TRAINING-TEST SET DISTRIBUTIONS

\begin{tabular}{|c|c|c|}
\hline Training & Testing & Accuracy (\%) \\
\hline 10 & 96 & 54.5 \\
\hline 20 & 86 & 59.2 \\
\hline 30 & 76 & 65.6 \\
\hline 40 & 66 & 72.2 \\
\hline 50 & 56 & 73.1 \\
\hline 60 & 46 & 79.3 \\
\hline 70 & 36 & 84.1 \\
\hline 80 & 26 & 88.0 \\
\hline 90 & 16 & 88.9 \\
\hline 100 & 6 & 98.3 \\
\hline
\end{tabular}

sification results obtained using the graph-based ROIBIC approach reported in [6]. The graph based approach used a support threshold to identify frequent sub graphs and thus a range of support thresholds is presented in Table II. The graph based approach generated a sequence of quad trees, one per image; the algorithm allowed the user to pre-define the desired "granularity" of the representation by specifying the maximum number of levels in the generated quad trees. Hence Table II also includes results for different sized quad trees. The best result for each level is indicated in bold font.

Inspection of Tables I and II demonstrate that the overall classification accuracy of the ROIBIC time series approach significantly improves on the classification accuracy obtained using the graph based approach.

Image segmentation and the application of DTW for classification are both computationally expensive processes. The time complexity for the image segmentation was about 30 seconds per image. For the given data set the application of DTW for classification took 60 seconds on average to categorize the test set. Regarding the graph based approach, the computational complexity of the proposed approach to ROIBIC is low compared to the computational complexity of the graph based approach (which entailed significant overheads).

For completeness Table III shows the classification results obtained with time series based ROIBIC using different training-test set distributions. The "training" and "testing" columns give the training dataset and the testing dataset sizes respectively. As might be expected the accuracy of the classification improves with the size of the training set indicating the benefits that might be attained if larger training sets were available.

\section{SUMMARY AND CONCLUSION}

In this paper an approach to Region Of Interest Based Image Classification (ROIBIC) founded on time series analysis 
has been described. The work was directed at a particular MRI scan classification application, the classification of MRI scans according to the nature of the corpus callosum featured within these images. However, the approach has more general applicability. Of particular note is the high classification accuracy obtained using the ROIBIC time series approach with respect to the corpus callosum application. Currently the research team are working on the more general application of the technique, such as the automated identification of disorders such as Epilepsy from MRI data. The research team are also interested in alternative methods of pre-processing MR image data, and mechanism for the post-processing of results to provide explanations for specific classifications. The latter is seen as particularly significant in the context of medical research involving MRI scan data.

\section{REFERENCES}

[1] R. Chen and E. Herskovits, "A Bayesian Network Classifier with Inverse Tree Structure for Voxelwise Magnetic Resonance Image Analysis," Proc. ACM SIGKDD 2005, 2005, pp 4-12.

[2] Y. Zhang, L. Wu, N. Neggaz, S. Wang and G. Wei, "Remote-Sensing Image Classification Based on an Improved Probabilistic Neural Network," Sensors, vol. 9, no. 9, pp. 7516-7539, 2009.

[3] C. Shyu, C. Pavlopoulou, A. Kak, C. Brodley and L. Broderick, "Using Human Perceptual Categories for Content-Based Retrieval from a Medical Image Database," Computer Vision and Image Understanding, vol. 88, no. 3, pp. 119-151, 2002.

[4] P. Felzenszwalb, and D. Huttenlocher, "Efficient Graph-Based Image Segmentation," Int. Journal of Computer Vision, vol. 59, no. 2, pp. 167-181, 2005.

[5] D. Berndt and J. Clifford, "Using dynamic time warping to find patterns in time series," AAAI-94 workshop on Knowledge Discovery in Databases, Seattle, Washington, 1994, pp. 359 - 370.
[6] A. Elsayed, F. Coenen, C. Jiang, M. García-Fiñana and V. Sluming, "Corpus Callosum MR Image Classification," Proc. AI'2009, Springer, 2009, pp. 333-346.

[7] F. Florea, E. Barbu, A. Rogozan, A. Bensrhair and V. Buzuloiu, "Medical Image Categorization using a Texture Based Symbolic Description,' proc. IEEE International Conference on Image Processing, 2006, pp. 1489-1492.

[8] J. Shotton, M. Johnson and R. Cipolla, "Semantic texton forests for image categorization and segmentation," proc. IEEE Conference on Computer Vision and Pattern Recognition, CVPR 2008, 2008, pp. 1-8.

[9] S. Park, J. Lee and S. Kim, "Content-based image classification using a neural network," Pattern Recognition Letters, vol. 25, no. 3, pp. 287300, 2004

[10] S. Saha, and R. Vemuri "Image categorization and coding using neural networks and adaptive wavelet filters," Proc. of IEEE International Conference on Industrial Technology 2000, 2000, pp. 438-443.

[11] O. Chapelle, P. Haffner and V. Vapnik, "Support vector machines for histogram-based image classification," IEEE Transactions on Neural Networks, vol. 10, no. 5, pp. 1055-1064, 1999.

[12] A. Jiang, C. Wang and Y. Zhu, "Calibrated Rank-SVM for multi-label image categorization," IEEE International Joint Conference on Neural Networks. IJCNN 2008, 2008, pp. 1450-1455.

[13] E. Keogh and M. Pazzani, "Scaling up dynamic time warping to massive datasets," Proc. of the 3rd Europ. Conf. on Principles of Data Mining and Knowl. Discovery,1999, pp. 1-11.

[14] C. Ordonez and E. Omiecinski, "Discovering Association Rules Based on Image Content," Proc. IEEE Advances in Digital Libraries Conference, 1999 , pp. 38-49.

[15] H. Pan, J. Li and Z. Wei, "Mining Interesting Association Rulers in Medical Images," Proc. ADMA'05, LNAI 3584, Springer-Verlag, 2005, pp. 598-609

[16] X. Burgos-Artizzu, A. Ribeiro, A. Tellaeche, G. Pajares and C. Fernndez-Quintanilla, "Improving weed pressure assessment using digital images from an experience-based reasoning approach," Computers and Electronics in Agriculture archive, vol. 65, no. 2, pp. 176-185, 2009.

[17] M. Grimnes and A. Aamodt, "A Two Layer Case-Based Reasoning Architecture for Medical Image Understanding," Proc. of the Third European Workshop on Advances in Case-Based Reasoning, 1996, pp. 164-178.

[18] R. Macura and K. Macura, "MacRad: Radiology Image Resource with a Case-Based Retrieval System," Proc. of the First International Conference on Case-Based Reasoning Research and Development, 1995, pp. 43-54. 